\title{
ANN Based Model for Estimation of Transformation Hardening of AISI 4340 Steel Plate Heat-Treated by Laser
}

\author{
Guillaume Billaud, Abderazzak El Ouafi, Noureddine Barka \\ Mathematics, Computer Science and Engineering Department, University of Quebec at Rimouski, Rimouski, \\ Canada \\ Email: abderrazak_elouafi@uqar.ca
}

Received 7 October 2015; accepted 15 November 2015; published 18 November 2015

Copyright (C) 2015 by authors and Scientific Research Publishing Inc.

This work is licensed under the Creative Commons Attribution International License (CC BY). http://creativecommons.org/licenses/by/4.0/

(c) (i) Open Access

\begin{abstract}
Quality assessment and prediction becomes one of the most critical requirements for improving reliability, efficiency and safety of laser surface transformation hardening process (LSTHP). Accurate and efficient model to perform non-destructive quality estimation is an essential part of the assessment. This paper presents a structured and comprehensive approach developed to design an effective artificial neural network (ANN) based model for quality estimation and prediction in LSTHP using a commercial $3 \mathrm{~kW}$ Nd:Yag laser. The proposed approach examines laser hardening parameters and conditions known to have an influence on performance characteristics of hardened surface such as hardened bead width (HBW) and hardened depth (HD) and builds a quality prediction model step by step. The modeling procedure begins by examining, through a structured experimental investigations and exhaustive 3D finite element method simulation efforts, the relationships between laser hardening parameters and characteristics of hardened surface and their sensitivity to the process conditions. Using these results and various statistical tools, different quality prediction models are developed and evaluated. The results demonstrate that the ANN based assessment and prediction proposed approach can effectively lead to a consistent model able to accurately and reliably provide an appropriate prediction of hardened surface characteristics under variable hardening parameters and conditions.
\end{abstract}

\section{Keywords}

Laser Hardening Process, AISI 4340 Steel, Case Depth, Hardened Bead Width, Artificial Neural Network 


\section{Introduction}

In the industry, many steel components require a surface heat treatment in order to have the desired surface qualities such as hardness and wear resistance. Among the available processes, laser hardening process (LHP) is one of the most efficient, as it allows a very fast and localized metallurgical transformation. In addition, the process generates a hard surface layer with low distortion [1]. Using high energy beam, the surface is rapidly heated to reach the transition temperatures (microstructure changes) before being quenched by heat conduction into the colder core of the material. Consequently, a martensitic layer is produced without affecting the core of the material [2].

Despite its industrial advantages, predicting hardness profiles with a good accuracy remains difficult. Indeed, besides the process parameters (Power, scanning velocity and focus diameter), which can be properly set, the process is affected by the non-linear behavior of thermo-physical and metallurgical properties of the material [1]. It makes the temperature distribution uneasy to predict by complicating the resolution of the governing heatflow equation. Experimental tests are also expensive in terms of time and resources, especially if one wants to test many combinations of control parameters to have a better understanding of the process.

Among all the approaches that can be used to understand the process and ultimately to predict its performance, 3D simulation represents a powerful tool for combining multi-physics problems and taking into account the material and complex geometries. In fact, the developed model includes the non-linear properties of the material and the heat-flow equation is solved using the finite element method (FEM) [3] [4]. The FEM enables solving the governing heat-flow equation that determines the temperature distribution for each time step during the heating process. The hardness is then approximated by the equations of Ashby and Easterling [5]. The advantage of the simulation is that, although it might be long and tedious to implement, once it is completed and experimentally verified in a few cases, one can test any combination of input parameters and quickly generate a large number of data which can be used for further exploitation. Many studies have been conducted to optimize the various laser process parameters (surface hardening, laser welding, laser cutting, etc.) through statistical methods such as the ANOVA method. It can be applied in many fields of engineering, including production processes and products for professional and consumer markets all over the world [6]. S-L Chen and D. Shen [7] used the Taguchi tools such as graphic designs of parameters and analysis of variance (ANOVA) to optimize the hardened depth (HD) and the hardened bead width (HBW) in the case of the LHP. Badkar and Pandey [8] used the same tools to determine the relative importance of each parameter on the LHP. K.Y. Bentounis, A.G. Olabi and M.S.J. Hashmi [9] conducted a similar study in the case of a laser welding process. Most recently, Sathiya et $a l$. [10] also used the Taguchi method to optimize the laser welding parameters. Given that experimental characterization requires great efforts in terms of time and money, it is not easy to experiment all the combinations of the input parameters. The Taguchi method really is an asset, as it is a partial factorial design that only requires some combinations of the input parameters in order to be performed and yet, it gives accurate statistical results in the overall process.

Others studies are conducted using artificial neural networks (ANN) in order to improve the performance of the laser processes [11]. Ciurana J. et al. [12] used ANNs to establish a model for laser micromachining of hardened steel and to optimize the process parameters. Pan Q.Y et al. [13] performed a similar study by using a neural network to model the non-linear relationship between laser processing parameters and corrosion resistance of the surface of stainless steel during the process of laser surface re-melting, which locally improved the corrosion resistance of the steel. Munteanu and Adriana [14] predicted the surface hardness of steel using a neural network in the case of an electron beam machining process which is similar to the LHP. F. Lambiase et al. developed a prediction model of laser hardening by means of an ANN using experimental datasets and linear interpolations between those experimental measures to train the network. However, to obtain good and efficient modeling results with ANN techniques, a large quantity of experimental data is advantageous and the observations should cover a sampling space as wide as possible in order to simplify the interpolation task.

Indeed, in any modelling experiment, the results depend, to a large degree, on the method used to collect data. In a lot of cases, full factorial experiments are conducted. This approach cannot be implemented when too many factors are under consideration, because the number of repetitions required would be prohibitive in time and cost. Regular fractional factorial designs cannot produce credible results when interactions among the factors exist. By contrast, the use of a testing strategy such as the orthogonal arrays (OAs) developed by Taguchi leads to an efficient and robust fractional factorial design of experiments that can collect all the statistically significant data 
with a minimum number of repetitions. Accordingly, OAs are used in this study for the experimental design. On the other hand, by using 3D FEM simulation that can provide results matching fairly well with the experimentally observed variables, one can easily and quickly obtain additional data for any combination of input parameters. The quantity of simulated data generated in a short time compared to experimental data would allow exhaustive statistical analysis including all levels of all input parameters. Moreover, with a large quantity of data for training, a simple Multilayer Perceptron ANN can be appropriate for modeling.

The objective of this paper is to present a structured and comprehensive approach developed to design an effective artificial neural network (ANN) based model for quality estimation and prediction in LSTHP using a commercial laser source. The proposed approach examines laser hardening parameters and conditions known to have an influence on performance characteristics of hardened surface such as hardened bead width (HBW) and hardened depth (HD) and builds a quality prediction model step by step. The modeling procedure begins by examining, through a structured experimental investigations and exhaustive 3D FEM simulation efforts, the relationships between laser hardening parameters and characteristics of hardened surface and their sensitivity to the process conditions. Using these results and various statistical tools, different quality prediction models are developed and evaluated. In order to carry out the models building procedure, an efficient modeling planning method combining neural networks paradigm, a multi-criteria optimization and various statistical tools is adopted.

\section{3D Model Implementation and Validation}

\subsection{Implementation}

The 3D FEM model is developed on the commercial software to estimate the temperature profiles. These temperature profiles are used to approximate the surface hardness profiles (surface hardness, HD, HBW). The part is a $50 \mathrm{~mm} \times 30 \mathrm{~mm} \times 5 \mathrm{~mm}$ parallelepiped (Figure 1).

In this study, the heat flux used for the simulation is considered as a Gaussian beam distribution type which is given by Equation (1) [1],

$$
E=E_{0} \times \exp \left\{-\left(\left[x-\left(x_{0}+V \times t\right)\right]^{2} /\left(2 w^{2}\right)+\left[y-y_{0}\right]^{2} /\left(2 w^{2}\right)\right)\right\}
$$

where $V$ is the scanning velocity, and $x_{0}$ and $y_{0}$ are the beam center coordinates at $t=0 \mathrm{~s}$. E $\left(\mathrm{W} / \mathrm{m}^{2}\right)$ represents a Gaussian heat flux moving according to the x-axis at the velocity V. $E_{0}$ is defined by Equation (2),

$$
E_{0}=P(1-R c) /\left(\pi w^{2}\right)
$$

where $w$ is the Gaussian beam radius, $P$ is the laser beam power, $R c$ is the reflection coefficient of the material surface [2].

The moving isothermal contours can be observed in Figure 2. Because of the Gaussian form of the beam, the temperature is at its maximum (about $1110 \mathrm{~K}$ ) at the center of the spot. The temperature decreases rapidly with

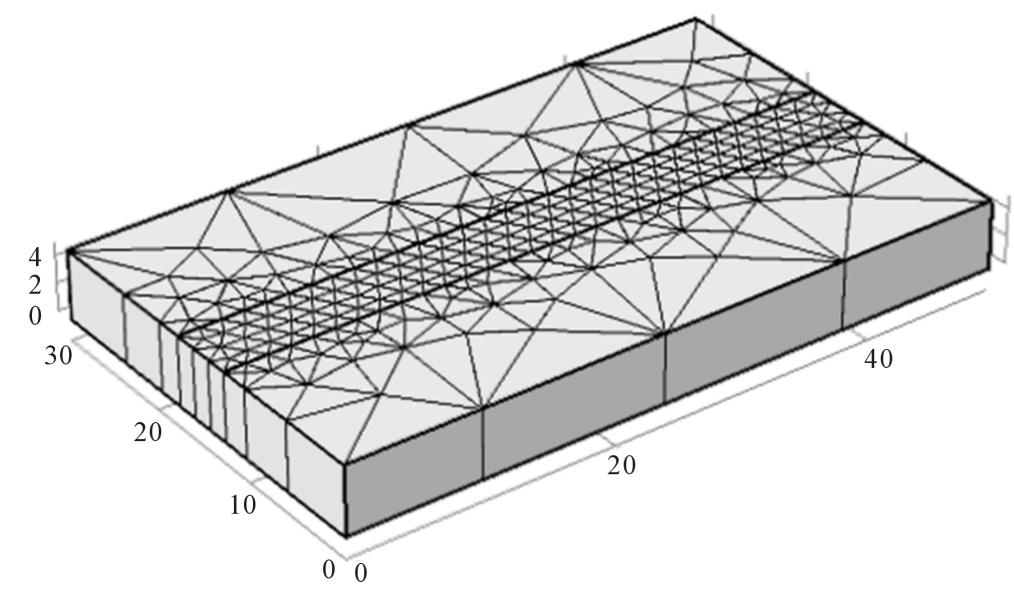

Figure 1. Sample with its mesh implemented on COMSOL. 


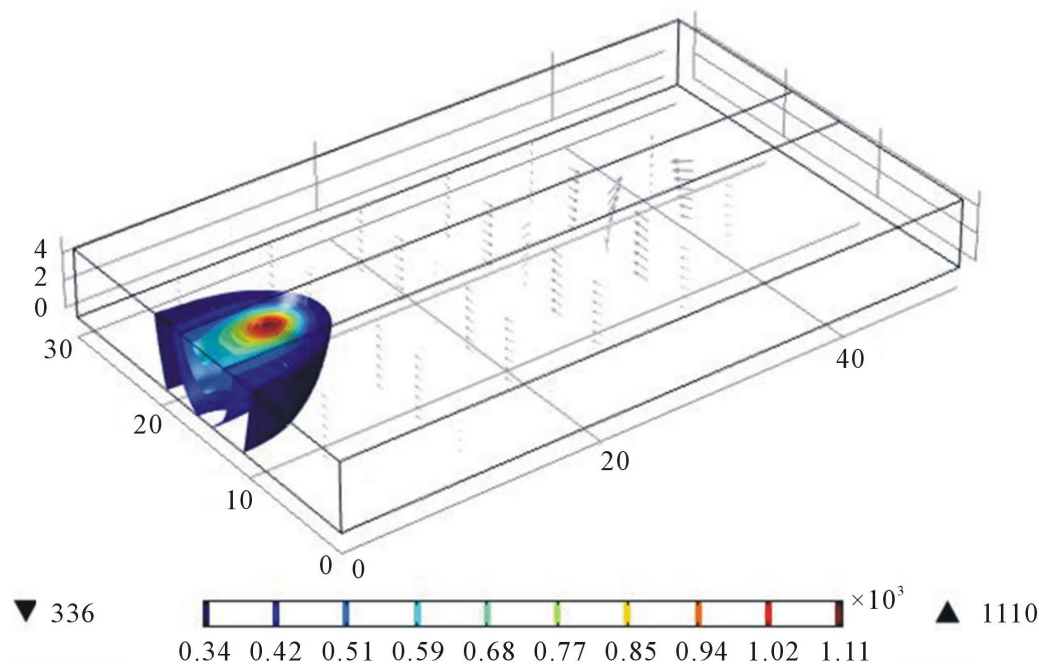

(a)

Isosurface: Temperature (K) Arrow Volume: Temperature gradient

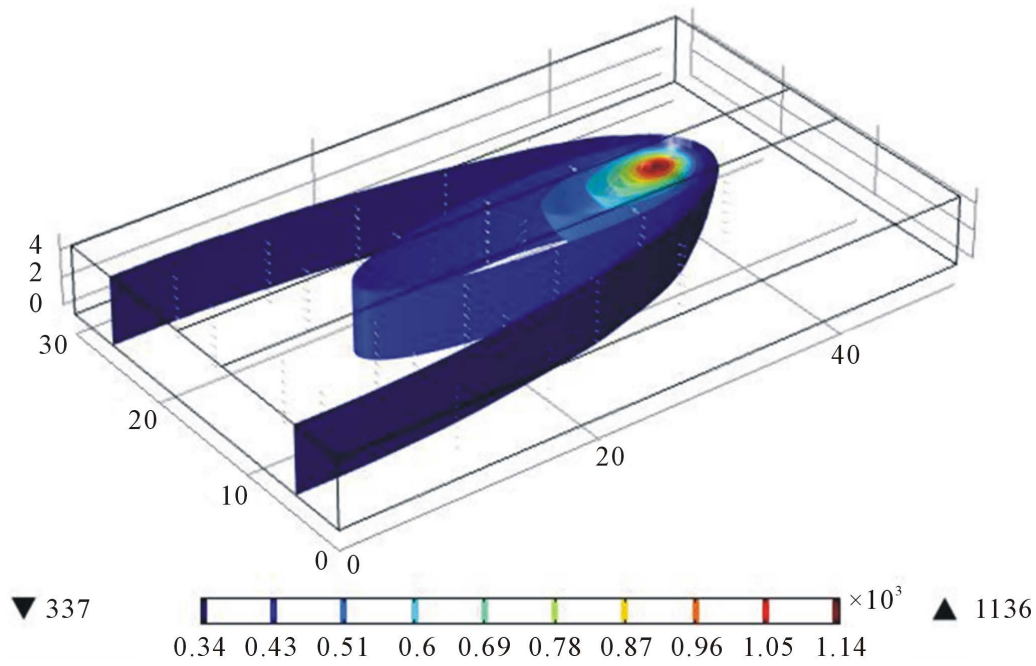

(b)

Figure 2. Isothermal contours: (a) $t=0.4 \mathrm{~s}$ and (b) $t=2.5 \mathrm{~s}$.

the depth because of heat conduction into the colder core of the material. The heated volume is small at the beginning of the process $(t=0.4 \mathrm{~s})$ and it gets larger as the time passes and the beam moves $(t=2.5 \mathrm{~s})$. As it can be seen in Figure 2, the small volume of the part that had reached the temperature of $1110 \mathrm{~K}$ at $\mathrm{t}=0.4 \mathrm{~s}$ (see Figure 2(a)) cool downed to reach a temperature under $430 \mathrm{~K}$ at $\mathrm{t}=2.5 \mathrm{~s}$ (see Figure 2(b)). It means that a very fast quenching happened in that volume.

Once the temperature distribution is determined, the hardness profile is estimated using the equations of Ashby and Easterling [5]. Those equations are implemented in MATLAB ${ }^{\circledR}$ to obtain the hardness at any point belonging to the heated part and, consequently, the hardness curve representing the hardness versus depth.

The 4340 steel properties are displayed in Table 1.

The specific heat and the thermal conductivity are temperature dependant and their dependency is taken into account in our model. 
Table 1. Metallurgical properties.

\begin{tabular}{cccc}
\hline Property & Symbol & Unit & Value \\
\hline Reflection coefficient & $R c$ & $\mathrm{~K}$ & 9.6 \\
Eutectoid temperature & $A c 1$ & $\mathrm{~K}$ & 1053 \\
Austenitization temperature & $A c 3$ & $\mu \mathrm{m}$ & 10 \\
Austenite grain size (assumed) & $g$ & $\mathrm{~kJ} / \mathrm{mol}$ & 80 \\
Activation energy of carbon diffusion in ferrite & $Q$ & $\mathrm{~m} / \mathrm{s}$ & $6 \times 10^{-5}$ \\
Pre-exponential for diffusion of carbon & $D 0$ & $\mathrm{~J} / \mathrm{mol} \cdot \mathrm{K}$ & 0.314 \\
Gas constant & $R$ & & $0.43 \%$ \\
Steel carbon content & $C$ & & $0.8 \%$ \\
Austenite carbon content & $C e$ & & $0.01 \%$ \\
Ferrite carbon content & $C f$ & & $0.05 \%$ \\
Critical value of carbon content & $C c$ & & 0.5375 \\
Volume fraction of pearlite colonies & fi & & \\
\hline
\end{tabular}

\subsection{Metallurgical Equations}

When the temperature in the material reaches the eutectoid temperature $\mathrm{Ac}_{1}$ in a small volume under the surface, the steel microstructure, which is generally tempered martensite in the case of the steel AISI 4340, starts to transform into austenite. The complete transformation from tempered martensite to austenite occurs when the temperature reaches $\mathrm{Ac}_{3}$. In the case of laser hardening treatment, when the temperature drastically decreases due to rapid heat diffusion into the colder core of the part, the austenite transforms into hard martensite. This is what is called a heat cycle (Figure 3).

As seen on Figure 3, the peak temperature at the surface is above $\mathrm{Ac}_{3}$. Therefore, a complete transformation into hard martensite happened at the surface. However, the peak temperature at $1.4 \mathrm{~mm}$ under the surface is under $\mathrm{Ac}_{1}$. It means that no transformation happened at this depth.

The total number of diffusive jumps that occur during the heat cycle affects the extent of the structural change and is given by the kinetic strength $I[1]$ [5],

$$
I=\int \exp \{-Q /(R \times T[t])\} \mathrm{d} t
$$

where $Q$ is the activation energy for the transformation and $R$ is the gas constant. It is more convenient to express $I$ as described in Equation (4).

$$
I=\alpha \tau \times \exp \left\{-Q /\left(R \times T_{p}\right)\right\}
$$

Here $T_{p}$ is the peak temperature at the considered depth and $\tau$ is the thermal time constant. The terms $\alpha$ and $\tau$ are approximated by Equations (5) and (6),

$$
\begin{gathered}
\alpha=3 \sqrt{\left[\left(R \times T_{p}\right) / Q\right]} \\
\tau=(1-R c) P /\left(2 \pi K e^{1} V\left[T_{p}-T_{0}\right]\right)
\end{gathered}
$$

where $T_{0}$ is the initial temperature.

The obtained austenite has the same carbon content as a perlite microstructure $C_{e}=0.8 \%$. From there, the carbon diffuses into the proeutectoid ferrite. When the temperature reaches $A c_{1}$, the volume fraction of austenite is $f_{i}$ (which is also the minimum volume fraction of martensite), given by Equation (7),

$$
f_{i}=\left(C-C_{f}\right) /\left(0.8-C_{f}\right) \approx C / 0.8
$$

where $C_{f}$ is the negligible carbon content of the ferrite and $C$ is the carbon content of the steel. 


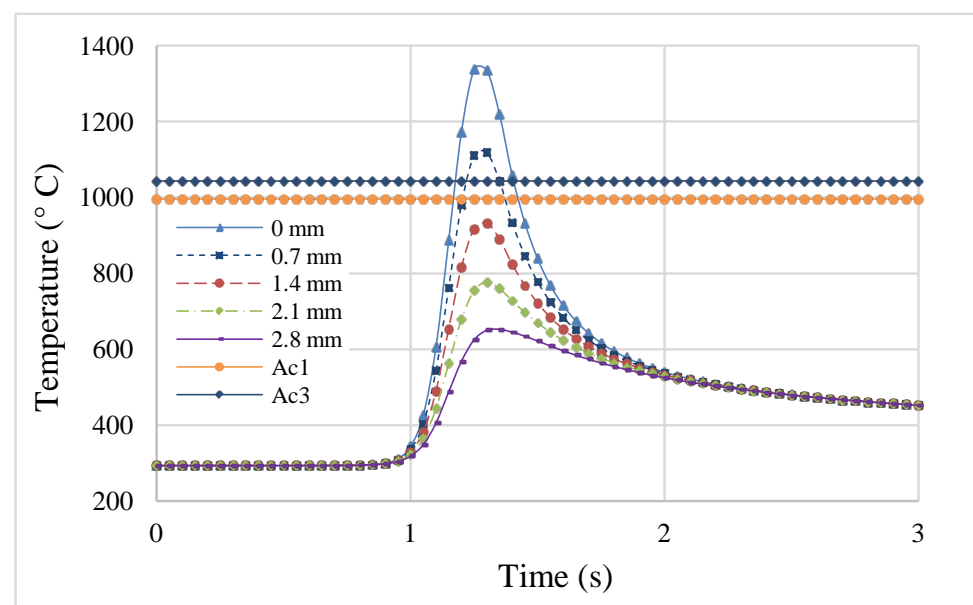

Figure 3. Temperature evolution at different depths (850 W and $9 \mathrm{~mm} / \mathrm{s})$.

The maximum martensite fraction allowed by the transformation temperature time diagram (TTT diagram) is

$$
\begin{aligned}
& \text { fm }=0 \AA \circledast \otimes \cap \circledast \otimes \quad \text { if } T_{p}<A c_{1} \\
& f m=f i+\left(1-f_{i}\right)\left(T_{p}-A c_{1}\right) /\left(A c_{3}-A c_{1}\right) \quad \text { if } A c_{1}<T_{p}<A c_{3}
\end{aligned}
$$

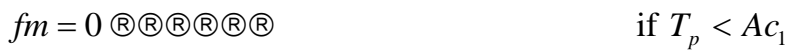

Ashby and Easterling supposed that all the material with a specific carbon proportion above the critical value $\mathrm{C}_{\mathrm{c}}$ will transform into martensite. The volume fraction of the martensite is then given by Equation (9) [1] [5].

$$
f=f m-\left(f m-f_{i}\right) \times \exp \left\{-\left(12 f_{i}^{2 / 3}\right) /(g \sqrt{\pi}) \times \ln \left[C_{e} /\left(2 C_{c}\right)\right] \sqrt{\left(D_{0} \times I\right)}\right\}
$$

Here $\mathrm{g}$ is the mean grain size and $D_{0}$ is the diffusion constant for the carbon in ferrite.

The hardness can then be calculated by a mixture rule (Equation (10)).

$$
H=f \times H_{m}+(1-f) \times H_{f+p}
$$

The value $H_{m}$ and $H_{f+p}$ are given by Maynier equations that take in account the cooling rate and the composition of the material [15].

\subsection{Experimental Validation}

The experimental procedure consists of a first heat treatment in a furnace with a water quenching followed by a tempering at $640^{\circ} \mathrm{C}$ for 1 hour. The aim is to reach a homogeneous hardness of $440 \mathrm{HV}$ for all the samples. Then, a commercial $3 \mathrm{~kW}$ Nd:Yag laser power (IPG YLS-3000-ST2), combined with a 6 degrees of freedom articulated robot (Figure 4) is used to perform laser heating. The plan-parallel sample is put on a metal plate under the laser head. This type of laser generates a laser beam with a wavelength $\lambda=1064 \mu \mathrm{m}$. The process parameters are the input power, the scanning velocity and the focus diameter. In this study, the laser beam has a straight-line trajectory as seen in Figure 2. Finally, the resulting case depth is measured by micro indentation.

Experimental validations are conducted according the Table 2. The focus diameter is $1260 \mu \mathrm{m}$ for the three tests. The values are chosen so that the surface temperature reaches the austenite temperature $\mathrm{Ac}_{3}$ but does not hit the melting temperature (about $1450^{\circ} \mathrm{C}$ ).

A micro-hardness machine is used to characterize the hardness curve as a function of the depth. After the laser treatment, the samples are prepared and polished to reach adequate surface finish. The hardness is then measured by using a micro-hardness machine. The validation is conducted by micro indentation, with $100 \mu \mathrm{m}$ steps between consecutive Vickers marks on the surface along a vertical axis. The experimental results help to validate and calibrate the model. In this sense, the obtained results confirm the concordance between the experimental and simulated hardness curves. This suggests that even if the developed model is not able to accurately 


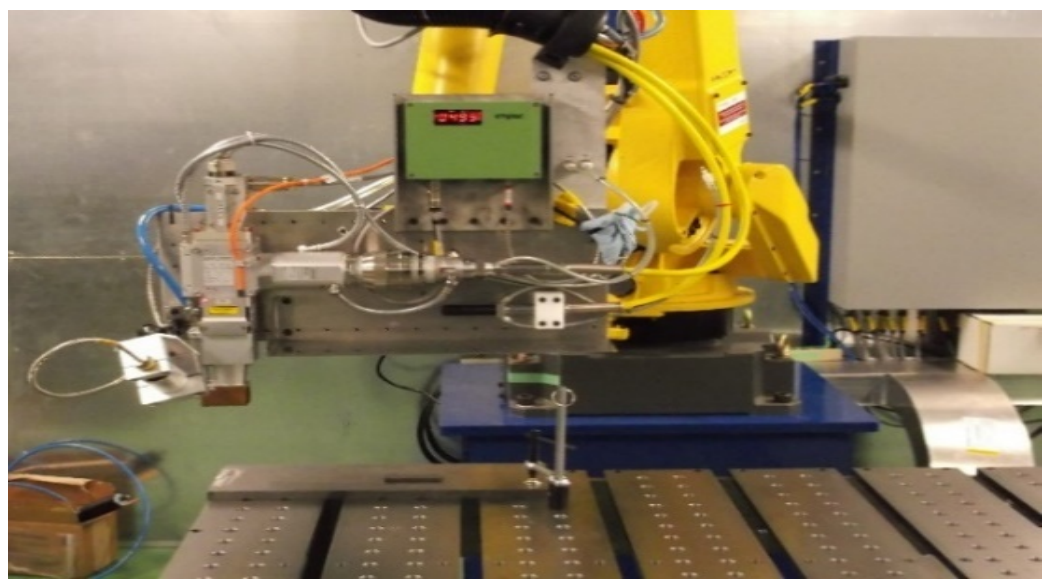

Figure 4. Experimental setup for model validation.

Table 2. Experimental matrix for validation.

\begin{tabular}{ccc}
\hline Test & Power $(\mathrm{W})$ & Scanning velocity $(\mathrm{mm} / \mathrm{s})$ \\
\hline 1 & 850 & 9 \\
2 & 850 & 12 \\
3 & 950 & 12 \\
\hline
\end{tabular}

predict the hardness curve, it can determine the hardened depth with good accuracy. Figures 5-7 show a comparison between the simulated and measured hardness curve using Vickers hardness scale $(\mathrm{VH})$ for the three tests (Table 2). It is worth noting that the developed 3D model is unable to predict the over-tempered zone where the hardness of the material becomes inferior to the initial hardness. However, hardened zone, transition zone and unaffected zone are correctly predicted. As expected, the hardened depth (at the start of the transition zone) increases as the power rises and/or the scanning velocity decreases. Table 3 shows the average absolute and relative errors between measured and simulated hardness. The preliminary tests allow to conclude that, despite the difference of more than $50 \mathrm{HV}$ in terms of absolute error, the relative error is very small, not exceeding 10\%. As shown in Figures 5-7, the simulation is fairly accurate in both hardness prediction and case depth prediction.

\section{Calibration of the Model with Corrected $\boldsymbol{R}_{c}$}

The coefficient Rc can be estimated around 0.6 for steel [2]. However, this coefficient greatly varies according to the surface temperature. Moreover, the surface temperature depends on the process parameters. In order to correctly calibrate Rc, different combinations of process parameters are executed using laser heating cell and the Rc is corrected so the results generated by the simulation match the experimental results for each set of input parameters. Finally, Rc is approximated as a function of the process parameters using a linear regression technique. Table 4 shows the Rc values depending on the laser power, the scanning velocity and the focus radius of the beam spot. The coefficient seems to increase as the power and/or the scanning velocity increases. Also, it seems to decrease when the focus radius increases.

The regression equation (Equation (11)) proves that there is a linear relationship between Rc and the process parameters. The correlation coefficient is 0.994 , which confirms a good correlation.

$$
\mathrm{Rc}=0.4205+0.000303 \times \mathrm{P}+0.003553 \times \mathrm{V}-0.000198 \times \mathrm{Rad}
$$

$\mathrm{P}$ is the input power in $\mathrm{W}, \mathrm{V}$ is the scanning velocity in $\mathrm{mm} / \mathrm{s}$, and Rad is the focus radius in $\mu \mathrm{m}$. The developed equation is incorporated in the simulation model. The HD and HBW can be estimated with good accuracy as a function of the process parameters. 


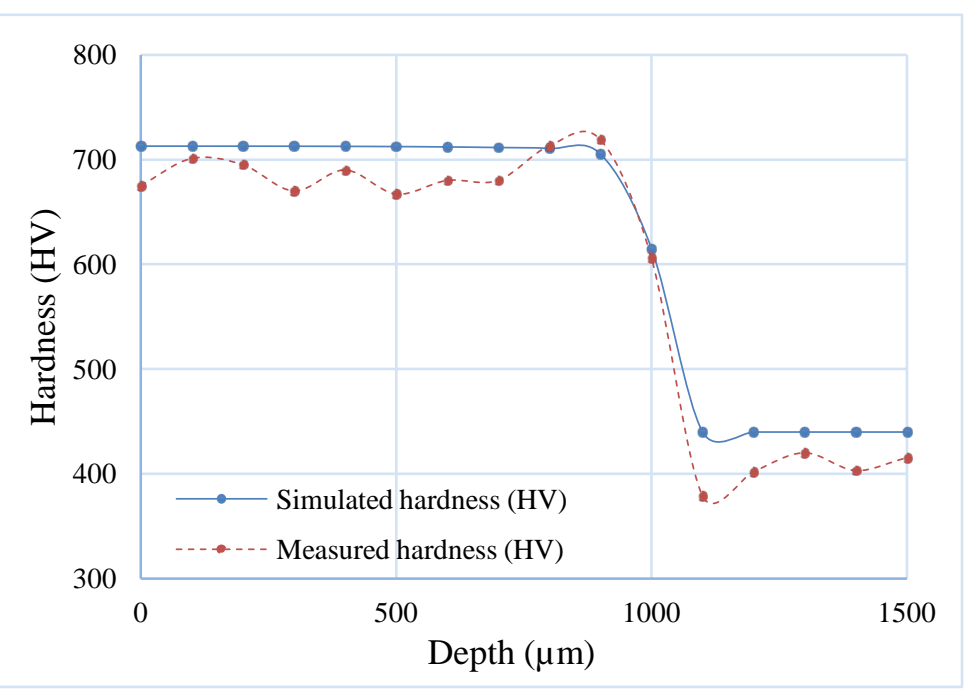

Figure 5. Hardness curve for test 1 ( $850 \mathrm{~W}$ and $9 \mathrm{~mm} / \mathrm{s}$ ).

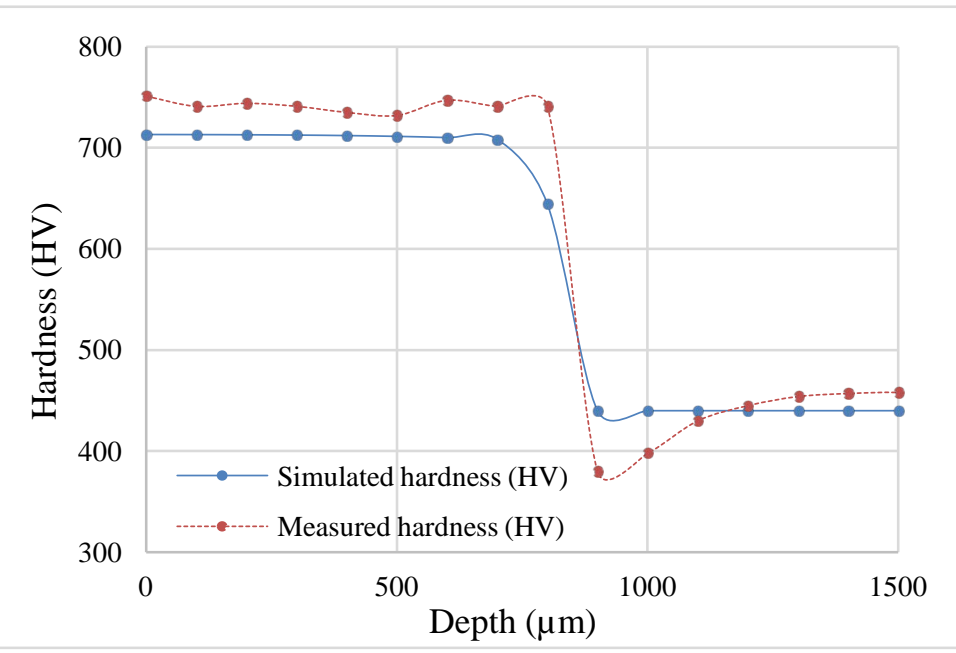

Figure 6. Hardness curve for test 2 (850 W and $12 \mathrm{~mm} / \mathrm{s}$ ).

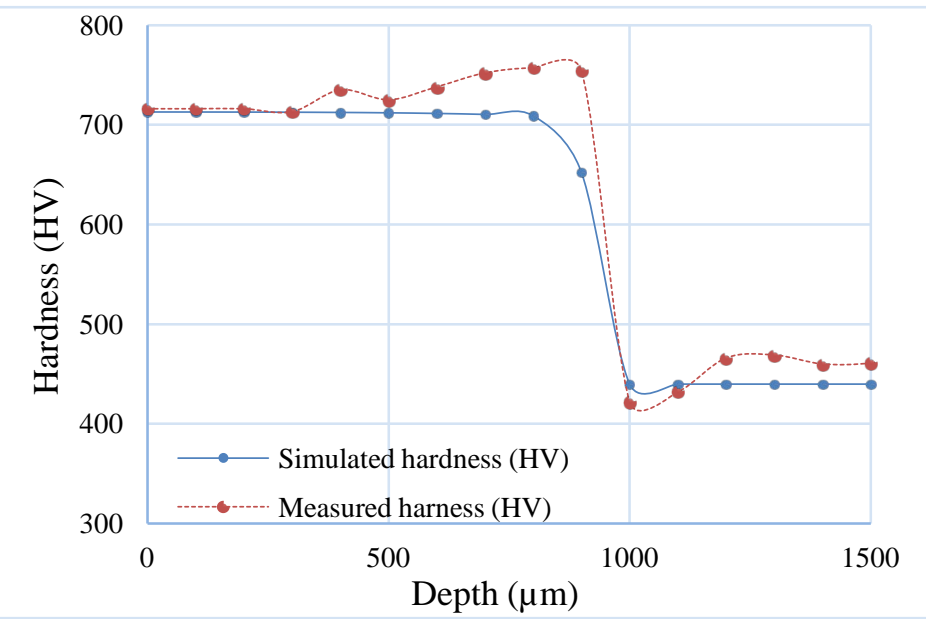

Figure 7. Hardness curve for test 3 (950 W and $12 \mathrm{~mm} / \mathrm{s}$ ). 
Table 3. Average absolute and relative hardness errors resulting from the preliminary tests.

\begin{tabular}{ccc}
\hline Test & Absolute error (HV) & Relative error (\%) \\
\hline 1 & 43 & 8.8 \\
2 & 30 & 5.2 \\
3 & 24 & 4.0 \\
\hline
\end{tabular}

Table 4. Corrected Rc according to process parameters.

\begin{tabular}{cccc}
\hline Power P $(\mathrm{W})$ & Scanning velocity V (mm/s) & Focus radius Rad $(\mu \mathrm{m})$ & Corrected Rc \\
\hline 400 & 20 & 550 & 0.50 \\
520 & 20 & 550 & 0.54 \\
630 & 20 & 550 & 0.61 \\
740 & 20 & 550 & 0.47 \\
400 & 12 & 550 & 0.49 \\
400 & 16 & 550 & 0.51 \\
400 & 16 & 480 & 0.48 \\
400 & 16 & 613 & 0.47 \\
\hline
\end{tabular}

\section{Shadowgraph Measurement}

As this study is focused on the HD and HBW and not the hardness values themselves, the depth and width are measured using optical method based on shadowgraph measurement. Figure 8 shows a micrographic picture of a part heat treated by laser with a power of $1000 \mathrm{~W}$ and a scanning velocity of $12 \mathrm{~mm} / \mathrm{s}$. The hardened region with hard martensite appears very clearly after a chemical treatment and can even be observed with the naked eye. Two significant zones can be distinguished. The first one is the melted region near the surface that received a great amount of energy, enough to reach the melting point. The second region represents the hardened region where the temperature exceeded the austenitization temperature $\left(\mathrm{Ac}_{3}\right)$ without reaching the melting point and where the microstructure changed into martensite upon self-quenching.

\section{Statistical Study}

In the present study, the objective is to predict the HD and HBW with given process parameters provided by the great number of data generated through simulation (assuming the input parameters are included in the range of study). A statistical study is conducted through a design of experiment (DOE) to determine the relative significance of each parameter and the interactions between them. The ANOVA method aims to study the effects of parameters on the hardness. It gives the contribution of each parameter on the variation of the outputs (HD and HBW). The process parameters and their design levels are displayed in Table 5. The levels are chosen so that the surface transformation happens and the surface temperature does not hit the melting temperature regardless of the combination of process parameters.

The simulation allowed us to quickly obtain results for all $64\left(4^{3}\right)$ possible combinations of factor levels, and thus to generate a full factorial design.

Statistical studies such as analysis of variance, main effects studies and linear regression are conducted.

\subsection{ANOVA for HD versus $P, V$ and Rad}

Table 6 presents the detailed statistical analysis. An F-value above 11.77 implies that the parameter is very significant. In this case, power (P), scanning velocity (V), focus radius (Rad) are all significant models terms. The interaction terms are less important since their contributions are less than $0.4 \%$. Also, it is clear that the power 


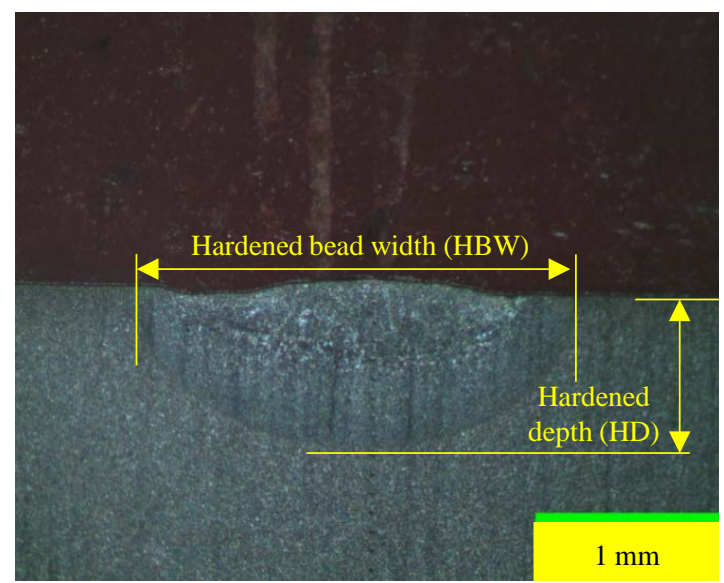

Figure 8. Micrographic picture illustrating the HBW and HD after chemical etching.

Table 5. Factors and levels used for the ANOVA study.

\begin{tabular}{ccccc}
\hline Factors & \multicolumn{5}{c}{ Factor Levels } \\
\hline Laser Power (P) [W] & 410 & 520 & 613 & 740 \\
Scanning Velocity (V) $[\mathrm{mm} / \mathrm{s}]$ & 12 & 16 & 18 & 20 \\
Focus Radius (Rad) $[\mu \mathrm{m}]$ & 480 & 550 & 613 & 663 \\
\hline
\end{tabular}

Table 6. ANOVA for HD.

\begin{tabular}{|c|c|c|c|c|c|c|}
\hline Source & DF & SS & contribution & MS & F-value & $\mathrm{p}$-value \\
\hline $\mathrm{P}$ & 3 & 563906 & $48.4 \%$ & 187969 & 499.71 & 0.000 \\
\hline V & 3 & 568906 & $48.8 \%$ & 189635 & 504.14 & 0.000 \\
\hline Rad & 3 & 13281 & $1.1 \%$ & 4427 & 11.77 & 0.000 \\
\hline $\mathrm{P} \times \mathrm{V}$ & 9 & 4531 & $0.4 \%$ & 503 & 1.34 & 0.264 \\
\hline $\mathrm{P} \times \mathrm{Rad}$ & 9 & 2656 & $0.2 \%$ & 295 & 0.78 & 0.632 \\
\hline $\mathrm{V} \times \mathrm{Rad}$ & 9 & 2656 & $0.2 \%$ & 295 & 0.78 & 0.632 \\
\hline Model & 36 & 1155936 & $99.1 \%$ & 383124 & & \\
\hline Error & 27 & 10158 & $0.9 \%$ & 376 & & \\
\hline Total & 63 & 1166094 & & & & \\
\hline
\end{tabular}

and the scanning velocity have the largest effect on the response value and that they are equivalent with contributions around $48 \%$. The three interaction terms can be considered negligible.

Figure 9 shows the effect of all parameters on the case depth (HD). The obtained results confirm that the HD increases as beam power increases and/or as scanning velocity decreases. It also increases as the focus radius decreases. The ANOVA method is conducted in order to assess the significance of each parameter. For each parameter studied, the variance ratio value, F, is compared to the values from standard F-tables for given statistical levels of significance. In this way, it is concluded that within the investigated processing ranges, the power, the scanning velocity and the focus radius are significant for the case depth at $95 \%$ confidence. Since the interaction terms have negligible contributions, they will not be considered in the rest of the study. Figure 10 shows the HD calculated using the regression formula (Equation (12)) for all 64 combinations of process parameters and their distribution around the bisector of the quadrant. If the formula is perfectly accurate, all the points should be on the bisector. For the regression formula to be considered accurate, a maximum relative error of $10 \%$ is allowed 

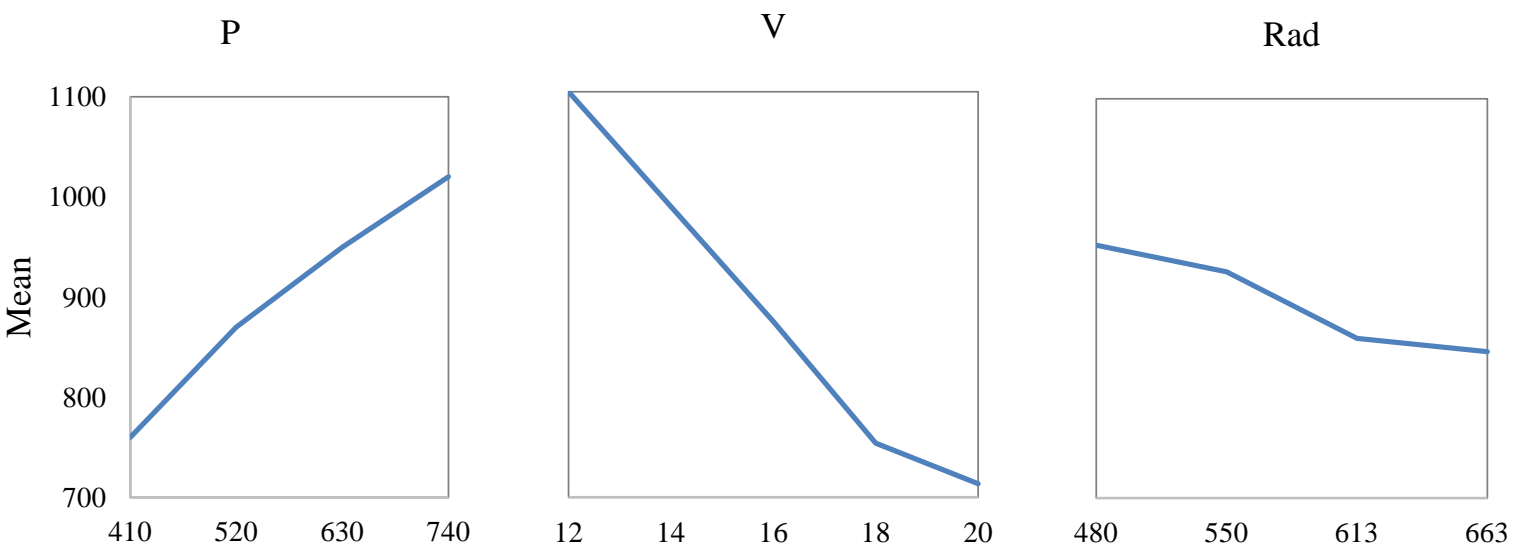

Figure 9. Effects of parameters on case depth.

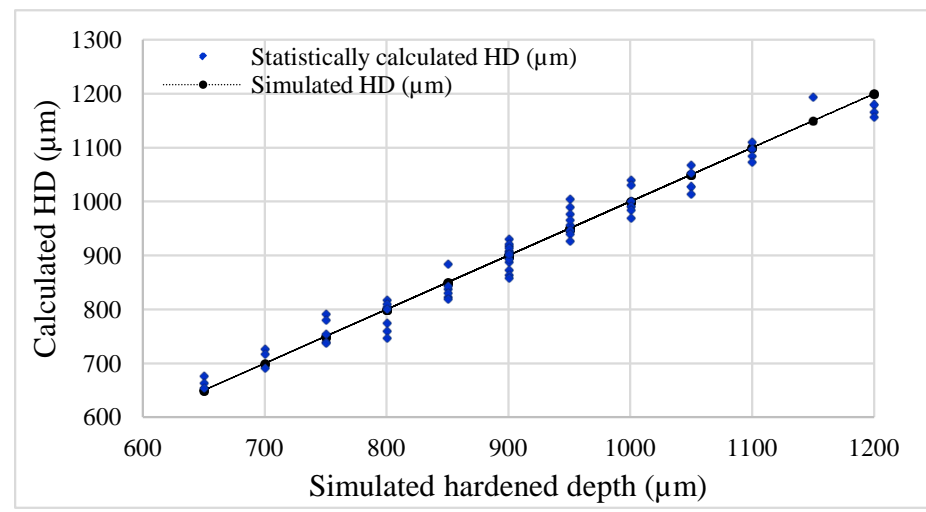

Figure 10. Comparison between simulated HD and HD calculated by regression formula (Equation (12)).

for all 64 sets of process parameters. A maximum relative error of $6.51 \%$ is observed, with a mean relative error of $2.25 \%$ between the HD calculated with the regression formula and the one simulated by the software. The coefficient of determination $\mathrm{R}^{2}$ is mainly used to measure the relationship between experimental data and measured data. A coefficient $\mathrm{R}^{2}=99.13 \%$ indicates an accurate study.

$$
\mathrm{HD}=1113.7+0.7557 \times \mathrm{P}-31.70 \times \mathrm{V}-0.2036 \times \mathrm{Rad}
$$

\subsection{ANOVA for HBW versus $P, V$ and Rad}

Table 7 shows the detailed statistical analysis. An F-value above 70.68 implies that the parameter is very significant. In this case, power (P), scanning velocity (V), focus radius (Rad) are all significant models terms. The interaction terms are less important since their contributions are less than $0.7 \%$. Also, it appears that the input power and the scanning velocity have the largest effect on the response value with contributions around $37 \%$ $43 \%$. The three interaction terms can be considered negligible. The coefficient of determination $\mathrm{R}^{2}$ is mainly used to measure the relationship between experimental data and measured data. Just like for the hardened depth, the input laser power and the scanning velocity have the same degree of impact (and the opposite effect); the other parameter (Rad) still have significance, and the interactions are negligible.

The first thing one can notice on Figure 11 is that the main effects plot for HBW is similar to the main effects plot for the HD, with the noticeable exception of the focus radius, which has the opposite effect on the HBW compared to the effect it has on the HD. Indeed, when the focus radius increases the HBW increases as well, whereas the HD decreases (see Figure 9 and Figure 11). This is caused by the Gaussian distribution of the energy at the surface of the material, which results in a relationship between HD and HBW. Indeed, the fact that the radius is greater while the power and the scanning velocity remain the same means that there will be less energy at the center of the focus. 
Table 7. Results of the ANOVA for HBW.

\begin{tabular}{ccccccc}
\hline Source & DF & SS & Contribution & MS & F-value & p-value \\
\hline P & 3 & 1763125 & $37.4 \%$ & 587,708 & 161.71 & 0.000 \\
V & 3 & 2023125 & $43 \%$ & 674,375 & 185.56 & 0.000 \\
Rad & 3 & 770625 & $16.4 \%$ & 256,875 & 70.68 & 0.000 \\
P $\times$ V & 9 & 13125 & $0.3 \%$ & 1458 & 0.40 & 0.923 \\
P $\times$ Rad & 9 & 30625 & $0.7 \%$ & 3403 & 0.94 & 0.511 \\
V $\times$ Rad & 9 & 10625 & $0.2 \%$ & 1181 & 0.94 & 0.959 \\
Model & 36 & 4611250 & $98 \%$ & 1525,000 & & \\
Error & 27 & 98125 & $2 \%$ & 3634 & & \\
Total & 63 & 4709375 & & & \\
\hline
\end{tabular}
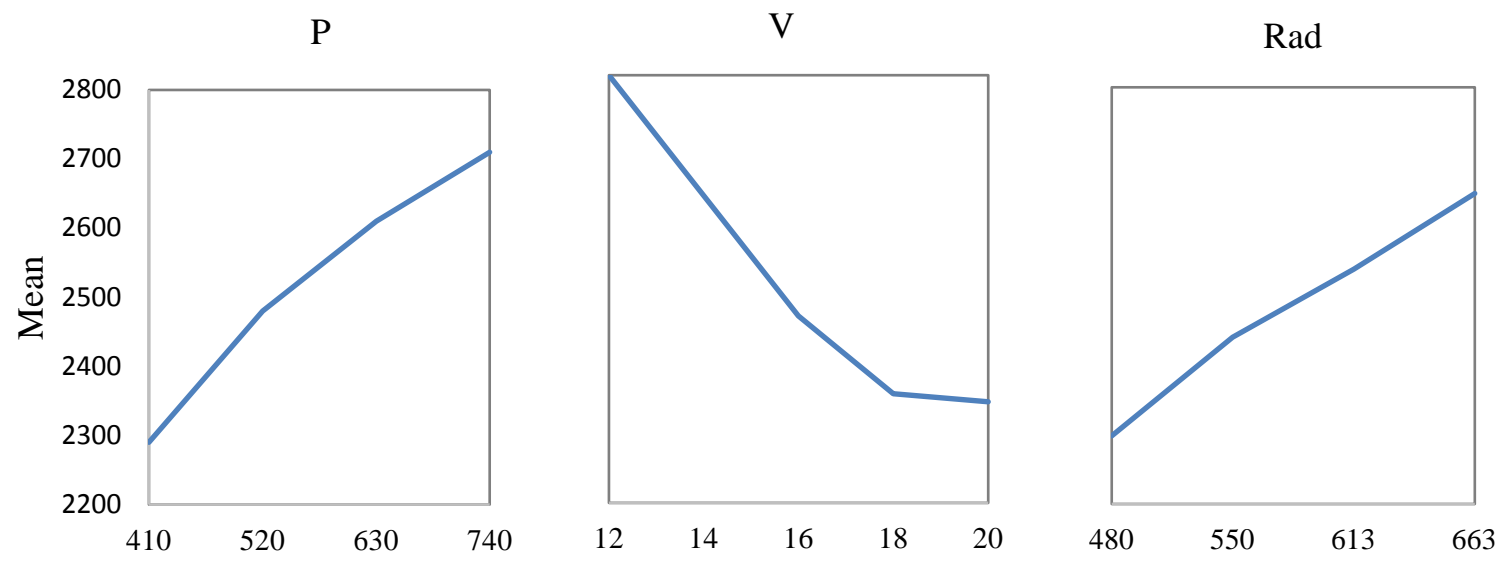

Figure 11. Main effects plot for hardened bead width.

As for the HBW, it appears that the interactions are negligible with very low F-value. Therefore they will not be included in the regression equation.

Figure 12 shows the HBW calculated using the regression formula (Equation (13)) for all 64 combinations of process parameters and their distribution around the bisector of the quadrant. If the formula is perfectly accurate, all the points should be on the bisector. A maximum relative error of $6.95 \%$ is observed, with a mean relative error of $2.39 \%$ between the HBW calculated with the regression formula and the one simulated by the software. Both values are well under the maximum criteria of $10 \%$ and thus, the formula can be considered accurate.

Moreover, the coefficient of determination $\mathrm{R}^{2}=97.92 \%$ testifies an accurate regression equation albeit not as satisfying as it is for the HD.

$$
\mathrm{HBW}=1782+1.3409 \times \mathrm{P}-57.68 \times \mathrm{V}+1.598 \times \mathrm{Rad}
$$

In addition to the statistical study, and in order to provide a reliable alternative to standard thermal techniques that would be accurate and less time consuming, we conducted a study with an artificial neural network (ANN).

\section{Neural Network Modeling}

As compared to other techniques, an ANN provides a more effective modeling capability, particularly when the relationship between sensor-derived information and the characteristic(s) to be identified is non-linear. ANNs can handle strong non-linearity, a large number of variables, and missing information. Based on their intrinsic learning capabilities, ANNs can be used in a case where there is no exact knowledge concerning the nature of the relationships between various variables. This is very useful in reducing experiment efforts. 


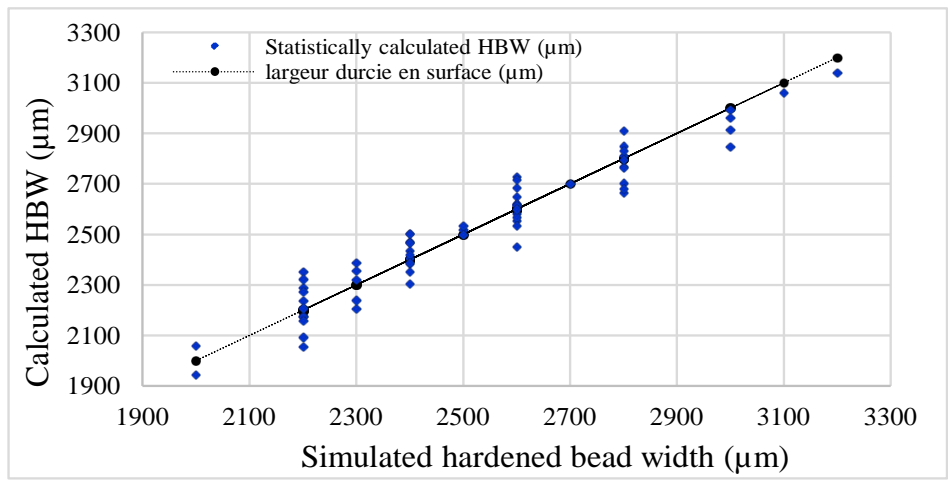

Figure 12. Comparison between simulated HBW and HBW calculated by regression formula (Equation (13)).

A neural network is used to predict the hardened depth and hardened bead width. Neural networks are generally presented as systems of interconnected neurons, where the links between neurons are weighted. Figure 13 shows the general principle of an ANN model. The goal is to produce one or more outputs that reflect the user-defined information stored in the connections during training.

In this study, a Generalized Feed-Forward Multilayer Perceptron (GFF-MLP) neural network model with one hidden layer containing 7 neurons is chosen. While various ANN techniques can be used in this approach, generalized feed forward networks seem to be the most appropriate because of their simplicity and flexibility. Before selecting the variables and training the models, it is important to establish the network topology and optimize the training performances. The idea is to approximate the relationship between the network parameters and the complexity of the variables to be estimated. The selected network is that which achieved the best results, the $[n|2 n+1| 3]$ network, where $n$ is the number of inputs. The perceptron is characterized by a nonlinear sigmoid function. This type of neural network is always fully connected, meaning each perceptron of each layer is connected with all the perceptrons in the previous layer [16]. In a GFF-MLP network, connections between layers can jump over one or more layers. In practice, these networks solve problems much more efficiently than MLP networks [17].

Neural networks need to be trained with data sets in order to be able to interpolate for any given input parameters that fall within the training range. Neural networks cannot extrapolate, which means one cannot get reliable outputs if the input parameters are not within the range of the training parameters. In this study, the goal is to obtain a neural network able to predict the case depth and hardened bead width for a given combination of input parameters (within its training range). In all neural networks, during the training step, the input data are normalized to the range of $[-1,1]$. The weights and biases of the network are initialized to small random values to avoid a fast saturation of the activation function.

\subsection{Maintaining the Integrity of the Specifications}

For a commercial laser device, there are usually 3 control parameters, the input power (P), the scanning velocity (V) and the focus radius (Rad). In this study, 4 levels for each of those parameters are chosen and are displayed in Table 5. The levels are chosen to ensure minimal martensitic transformation and to avoid the melting point (about $1450^{\circ} \mathrm{C}$ ) regardless of the combination of levels. With 3 parameters with 4 levels, the total number of possible combinations is $64\left(4^{3}\right)$. The simulation allows to quickly get all of the 64 combinations and produce a full L64 matrix as in the preceding statistical studies.

In addition to the training data, a neural network also requires verification data (that are different from the training data) in order to validate the training step. These verification data are displayed in Table 8. In this case, the mean value of two consecutive levels are identified and used in simulation to generate data for verification. This leads to a validation design of $3^{3}$ possible.

The neural network is trained considering the mean square error (MSE) of the cross-validation as an achievement indicator. The training of the neural network stops when the MSE stops decreasing. In order to evaluate the effectiveness of the network, some criteria are used, the correlation coefficient and the root mean square error, which would be respectively equal to 1 and 0 in the best case scenario with perfect accuracy. 


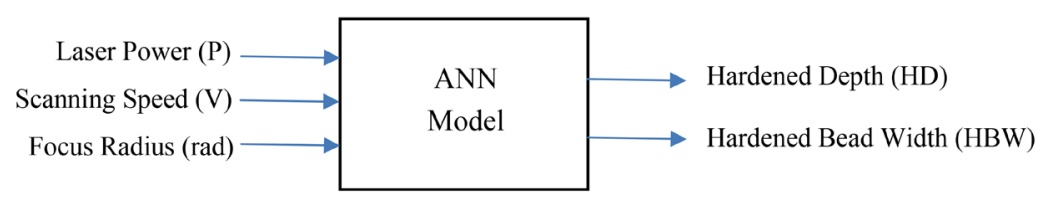

Figure 13. Principle of the neural network.

Table 8. Middle points.

\begin{tabular}{cccc}
\hline Factors & \multicolumn{3}{c}{ Factor Levels } \\
\hline Laser Power (P) $[\mathrm{W}]$ & 465 & 575 & 685 \\
Scanning Velocity $(\mathrm{V})[\mathrm{mm} / \mathrm{s}]$ & 14 & 17 & 638 \\
Focus Radius (Rad) $[\mu \mathrm{m}]$ & 515 & 581.5 & 6 \\
\hline
\end{tabular}

\subsection{Result and Interpretation}

Once the training step of the network is performed, the 27 combinations of verification data are applied as input parameters. The outputs of the ANN model are compared with those obtained by simulation. Therefore, this comparison is effective using various statistical indexes that characterize the prediction capability of the ANN model. Two main criteria are used to evaluate the accuracy of the network: the absolute error and the relative error.

Figure 14 shows the absolute errors for both HD and HBW for all 27 test combinations. The maximum absolute errors for HD and HBW are, respectively, 64 and $94 \mu \mathrm{m}$. This means that the absolute error is of less than $100 \mu \mathrm{m}$ for the overall test data, for both HD and HBW. Given that the values of HD are between $700 \mu \mathrm{m}$ and $1100 \mu \mathrm{m}$, and that the values of HBW are between $2400 \mu \mathrm{m}$ and $3000 \mu \mathrm{m}$, the model exhibits a good potential in terms of accuracy.

As can be seen in the Figure 15, the relative errors for both the HD and HBW are very low in every case. The maximum relative errors for HD and HBW are, respectively, $8.01 \%$ and $3.62 \%$. The mean relative errors for HD and HBW are $2.40 \%$ and $1.63 \%$, respectively, which heightens the accuracy of the neural network.

Figure 16 and Figure 17 present, respectively, the results of the ANN models during the verification stage for HD and HBW. In fact, the figures show the ANN model and those obtained by simulation. The data are mostly located around the bisector of the 1st quadrant, which outlines the accuracy of the model. The two figures show that the network is well trained and is highly efficient. The network is therefore a reliable way to predict the HD and HBW for any combination of input parameters within the training range (between $480 \mathrm{~W}$ and $663 \mathrm{~W}$ for power, $12 \mathrm{~mm} / \mathrm{s}$ and $20 \mathrm{~mm} / \mathrm{s}$ for scanning velocity, $480 \mathrm{~mm}$ and $663 \mathrm{~mm}$ for focus radius). The ANN models don't require any computation time to predict the outputs comparatively to the simulation. Note that the ANN models can predict the desired outputs in the studied variation range only and they cannot extrapolate outside.

Table 9 shows the comparison between the results generated by simulation and those generated by ANN model during training stage and confirms the observations from Figure 14 and Figure 15.

Even if the ANN models have good performances in terms of robustness and accuracy, it is still important to validate them using experimental validation.

\section{Experimental Validation of the Neural Network}

The great number of data that can be generated by a 3D FEM allows to accurately train a neural network that will be able to predict the HD and HBW, and thus, it avoids the need to produce expensive experimental data that are often less numerous because of their cost.

Once the network accuracy is verified with data generated by a FEM simulation, experimental validation tests are conducted using a Nd:Yag laser and the shadowgraph measurement method.

Eight sets are randomly chosen among the 27 sets of verification data. The experimental matrix is displayed in Table 10.

The input powers are between $465 \mathrm{~W}$ and $685 \mathrm{~W}$, the scanning velocities are between $14 \mathrm{~mm} / \mathrm{s}$ and $17 \mathrm{~mm} / \mathrm{s}$. Finally, the focus radii are between $515 \mu \mathrm{m}$ and $638 \mu \mathrm{m}$. 


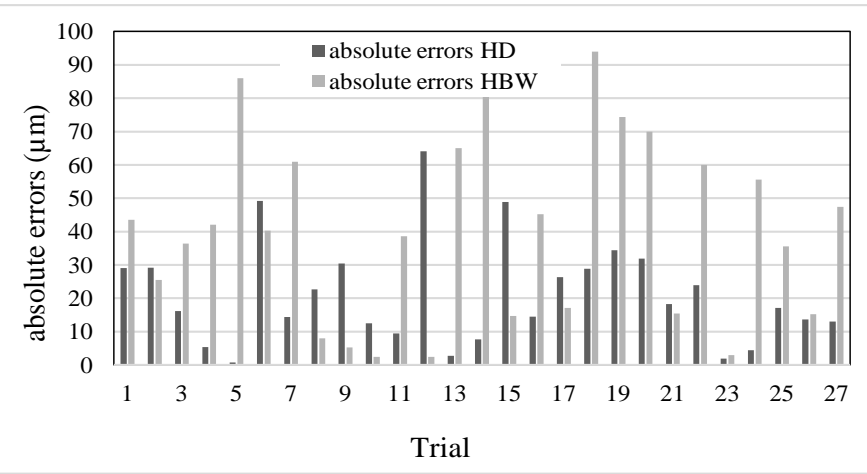

Figure 14. Absolute relative errors for HD and HBW.

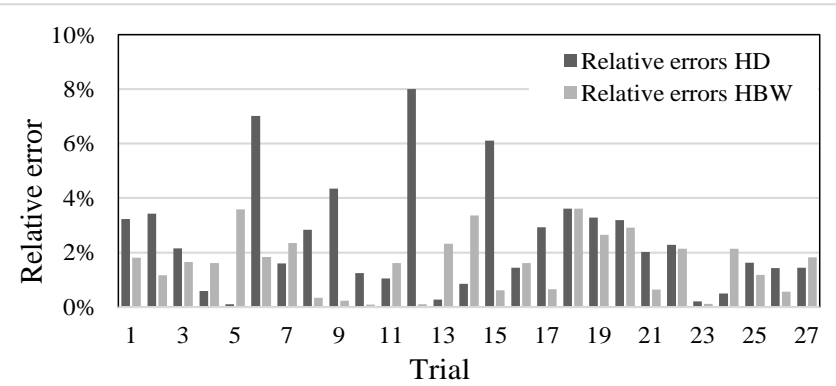

Figure 15. Relative errors for HD and HBW.

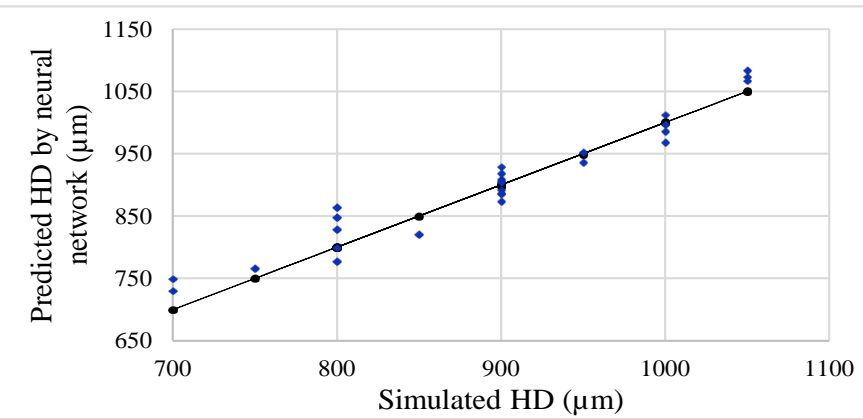

Figure 16. Comparison between simulated HD and HD calculated by the neural network.

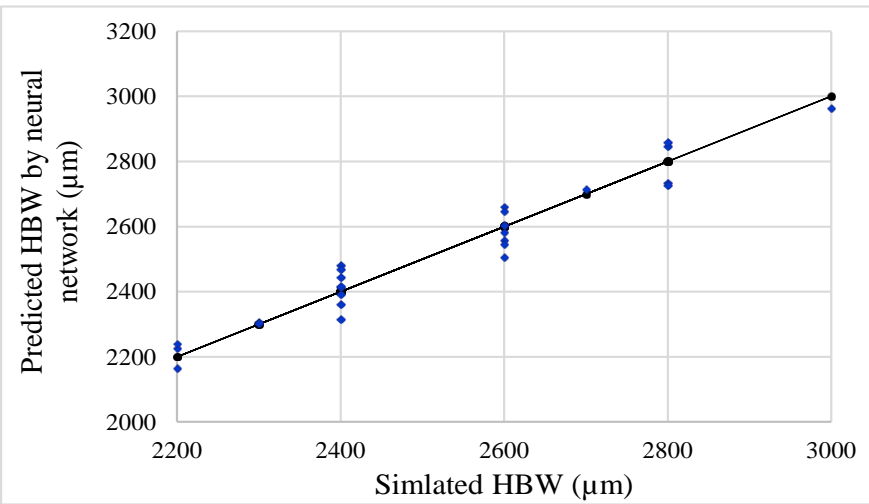

Figure 17. Comparison between simulated HBW and HBW calculated by the neural network. 
The results of the tests are shown in Table 11. The maximum relative errors for both the HD and HBW are $7.37 \%$ and $2.93 \%$, respectively.

The ANN is able to correctly predict both HD and HDW. It can now be used independently from the COMSOL software. It is easier to use as one only needs to compute the process parameters (within the training ranges of the ANN) to obtain reliable results instantly.

\section{Conclusion}

In this paper, a structured and comprehensive approach developed to design an effective ANN-based model for quality assessment and prediction in LSTHP using a commercial $3 \mathrm{~kW} \mathrm{Nd:Yag} \mathrm{laser} \mathrm{is} \mathrm{presented.} \mathrm{Several} \mathrm{laser}$ hardening parameters and conditions were analyzed and their correlation with multiple performance characteristics of hardened surface was investigated using a structured experimental investigations and exhaustive 3D FEM

Table 9. Comparison of the results.

\begin{tabular}{cccccccccc}
\hline & & \multicolumn{2}{c}{ Simulation } & \multicolumn{2}{c}{ ANN models } & \multicolumn{2}{c}{ Absolute error } & \multicolumn{2}{c}{ Relative error } \\
\cline { 3 - 10 } & & HD $(\mu \mathrm{m})$ & HBW $(\mu \mathrm{m})$ & HD $(\mu \mathrm{m})$ & HBW $(\mu \mathrm{m})$ & HD $(\mu \mathrm{m})$ & HBW $(\mu \mathrm{m})$ & HD $(\%)$ & HBW $(\%)$ \\
\hline \multirow{5}{*}{ Training } & Min & 700 & 2000 & 698 & 2058 & 2 & 58 & 0.03 & 0.05 \\
& Max & 1200 & 3200 & 1175 & 3147 & 25 & 53 & 4.88 & 4.52 \\
& Mean & 937 & 2557 & 931 & 2570 & 15 & 39 & 1.62 & 1.55 \\
& Min & 700 & 2200 & 730 & 2164 & 1 & 2 & 0.10 & 0.09 \\
& Merification & 1050 & 3000 & 1084 & 2964 & 64 & 94 & 8.01 & 3.62 \\
& Max & 894 & 2522 & 902 & 2521 & 21 & 40 & 2.40 & 1.63 \\
\hline
\end{tabular}

Table 10. Experimental matrix for validation.

\begin{tabular}{cccc}
\hline Test & Power $(\mathrm{W})$ & Scanning velocity $(\mathrm{mm} / \mathrm{s})$ & focus radius $(\mu \mathrm{m})$ \\
\hline 1 & 465 & 14 & 515 \\
2 & 575 & 19 & 515 \\
3 & 685 & 17 & 515 \\
4 & 685 & 14 & 515 \\
5 & 575 & 14 & 581.5 \\
6 & 685 & 17 & 581.5 \\
7 & 465 & 17 & 638 \\
8 & 685 & 17 & 638 \\
\hline
\end{tabular}

Table 11. Experimental validation-results.

\begin{tabular}{ccccccc}
\hline Test & $\begin{array}{c}\text { Network HD } \\
(\mu \mathrm{m})\end{array}$ & $\begin{array}{c}\text { Experimental HD } \\
(\mu \mathrm{m})\end{array}$ & $\begin{array}{c}\text { Relative error for } \\
\text { HD }(\%)\end{array}$ & $\begin{array}{c}\text { Network HBW } \\
(\mu \mathrm{m})\end{array}$ & $\begin{array}{c}\text { Experimental HBW } \\
(\mu \mathrm{m})\end{array}$ & $\begin{array}{c}\text { Relative error for } \\
\text { HBW }(\%)\end{array}$ \\
\hline 1 & 929 & 909 & 2.21 & 2444 & 2375 & 2.88 \\
2 & 864 & 894 & 3.35 & 2302 & 2320 & 0.76 \\
3 & 968 & 953 & 1.59 & 2470 & 2402 & 2.83 \\
4 & 1084 & 1010 & 7.37 & 2726 & 2653 & 2.74 \\
5 & 997 & 1018 & 2.04 & 2735 & 2702 & 2.22 \\
6 & 952 & 1022 & 6.86 & 2603 & 2591 & 2.46 \\
7 & 777 & 785 & 0.98 & 2392 & 2324 & 2.93 \\
8 & 936 & 1002 & 6.55 & 2715 & 2643 & 2.73 \\
\hline
\end{tabular}


simulations under consistent practical process conditions. Following the identification of the hardening parameters and conditions that provide the best information about the LSTHP operation, tow type of modeling techniques were proposed to assess and predict the hardened bead width and hardened depth (HD) of the laser transformation hardened AISI 4340 steel plate: multiple regression analysis and ANN approach. The results demonstrate that the regression approach can be used to achieve a relatively accurate predicting model with correlation larger than $90 \%$. The ANN models present greater results. The maximum relative errors for both HD and HBW are less than 8\% and 3\%, respectively. Globally, the performance of the ANN-based model for quality estimation and prediction in LSTHP shows significant improvement as compared to conventional methods. With a global maximum relative error less than $10 \%$ under various LSTHP conditions, the modeling procedure can be considered efficient and have led to conclusive results, due to the complexity of the analyzed process. The proposed approach can be effectively and gainfully applied to quality assessment for LSTHP, because it includes the advantages of ease of application, reduced modeling time and sufficient modeling accuracy.

\section{References}

[1] Kannatey-Asibu, E. (2009) Principles of Laser Materials Processing. Wiley, Hoboken. http://dx.doi.org/10.1002/9780470459300

[2] Steen, W.M. (2010) Laser Material Processing. 4th Edition, Springer, London. http://dx.doi.org/10.1007/978-1-84996-062-5

[3] Patwa, R. and Shin, Y.C. (2007) Predictive Modeling of Laser Hardening of AISI5150H Steels. International Journal of Machine Tools and Manufacture, 47, 307-320. http://dx.doi.org/10.1016/j.ijmachtools.2006.03.016

[4] Mioković, T., Schulze, V., Vöhringer, O. and Löhe, D. (2006) Prediction of Phase Transformations during Laser Surface Hardening of AISI 4140 including the Effects of Inhomogeneous Austenite Formation. Materials Science and Engineering: A, 435-436, 547-555. http://dx.doi.org/10.1016/j.msea.2006.07.037

[5] Ashby, M.F. and Easterling, K.E. (1984) The Transformation Hardening of Steel Surfaces by Laser Beams-I. HypoEutectoid Steels. Acta Metallurgica, 32, 1935-1948. http://dx.doi.org/10.1016/0001-6160(84)90175-5

[6] Antony, J. and Jiju Antony, F. (2001) Teaching the Taguchi Method to Industrial Engineers. Work Study, 50, $141-149$. http://dx.doi.org/10.1108/00438020110391873

[7] Chen, S.L. and Shen, D. (1999) Optimisation and Quantitative Evaluation of the Qualities for Nd-YAG Laser Transformation Hardening. International Journal of Advanced Manufacturing Technology, 15, 70-78. http://dx.doi.org/10.1007/s001700050041

[8] Badkar, D., Pandey, K. and Buvanashekaran, G. (2011) Parameter Optimization of Laser Transformation Hardening by Using Taguchi Method and Utility Concept. International Journal of Advanced Manufacturing Technology, 52, 10671077. http://dx.doi.org/10.1007/s00170-010-2787-z

[9] Benyounis, K.Y., Olabi, A.G. and Hashmi, M.S.J. (2005) Effect of Laser Welding Parameters on the Heat Input and Weld-Bead Profile. Journal of Materials Processing Technology, 164-165, 978-985. http://dx.doi.org/10.1016/j.jmatprotec.2005.02.060

[10] Sathiya, P., Abdul Jaleel, M.Y. and Katherasan, D. (2010) Optimization of Welding Parameters for Laser Bead-onPlate Welding Using Taguchi Method. Production Engineering-Research and Development, 4, 465-476. http://dx.doi.org/10.1007/s11740-010-0234-5

[11] Hagan, M.T., Demuth, H.B. and Beale, M.H. (1996) Neural Network Design: Pws Boston.

[12] Ciurana, J., Arias, G. and Ozel, T. (2009) Neural Network Modeling and Particle Swarm Optimization (PSO) of Process Parameters in Pulsed Laser Micromachining of Hardened AISI H13 Steel. Materials \& Manufacturing Processes, 24, 358-368. http://dx.doi.org/10.1080/10426910802679568

[13] Pan, Q.Y., Huang, W.D., Song, R.G., Zhou, Y.H. and Zhang, G.L. (1998) The Improvement of Localized Corrosion Resistance in Sensitized Stainless Steel by Laser Surface Remelting. Surface and Coatings Technology, 102, 245-255. http://dx.doi.org/10.1016/S0257-8972(98)00358-2

[14] Munteanu, A. (2012) Surface Hardness Prediction Using Artificial Neural Networks in Case of Electron Beam Machining Process. Romanian Association of Nonconventional Technologies.

[15] Doane, D. (1979) Application of Hardenability Concepts in Heat Treatment of Steel. Journal of Heat Treating, 1, 5-30. http://dx.doi.org/10.1007/BF02833206

[16] Haykin, S. (2010) Neural Networks: A Comprehensive Foundation, 1994. Mc Millan, New Jersey.

[17] Principe, J., Euliano, N. and Lefebvre, W. (2000) Neural and Adaptive Systems: Fundamentals through Simulations. John Wiley and Sons, New York. 